\title{
Symmetry reduction for dynamic programming and application to MRI
}

\author{
John Maidens, Axel Barrau, Silvère Bonnabel, and Murat Arcak
}

\begin{abstract}
We present a method of exploiting symmetries of discrete-time optimal control problems to reduce the dimensionality of dynamic programming iterations. The results are derived for systems with continuous state variables, and can be applied to systems with continuous or discrete symmetry groups. We prove that symmetries of the state update equation and stage costs induce corresponding symmetries of the optimal cost function and the optimal policies. Thus symmetries can be exploited to allow dynamic programming iterations to be performed in a reduced state space. The application of these results is illustrated using a model of spin dynamics for magnetic resonance imaging (MRI). For this application problem, the symmetry reduction introduced leads to a significant speedup, reducing computation time by a factor of $75 \times$.
\end{abstract}

\section{INTRODUCTION}

The dynamic programming algorithm for computing optimal control policies has, since its development, been known to suffer from the "curse of dimensionality" [1]. Its applicability in practice is typically limited to systems with five or six continuous state variables because the number of points required to grid a space of $n$ continuous state variables increases exponentially with the state dimension $n$. This complexity has led to a collection of algorithms for approximate dynamic programming, which scale to systems with larger state dimension but lack the guarantees of global optimality of the solution associated with the original dynamic programming algorithm [2]-[5].

In practice, many real-world systems exhibit symmetries that can be exploited to reduce the complexity of system models. Symmetry reduction has found applications in fields ranging from differential equations [6], [7] to model checking [8], [9]. In control engineering, symmetries have been exploited to improve control of mechanical systems [10], [11], develop more reliable state estimators [12], [13], study the controllability of multiagent systems [14] and to reduce the complexity of stability and performance certification for interconnected systems [15], [16]. Symmetry reduction has also been applied to the computation of optimal control policies for continuous-time systems in [17], [18] and Markov decision processes (MDPs) in [19], [20].

J. Maidens and M. Arcak are with the Department of Electrical Engineering \& Computer Sciences, University of California, Berkeley, 253 Cory Hall, Berkeley, CA, 94720 USA, e-mail: \{maidens, arcak\}@eecs.berkeley.edu. A. Barrau is with SAFRAN TECH, Groupe Safran, Rue des Jeunes Bois - Châteaufort, 78772 Magny Les Hameaux CEDEX, email: axel.barrau@mines-paristech.fr. S. Bonnabel is with MINES ParisTech, PSL Research University, Centre for Robotics, 60 bd Saint-Michel, 75006 Paris, France, email: silvere.bonnabel@mines-paristech.fr. Research supported in part by NSERC (Canada) postgraduate fellowship PGFD3-427610-2012, Amazon.com Inc. under and AWS Educate grant, and the National Science Foundation under grant ECCS-1405413.
In this paper we present preliminary results on symmetry reduction for the optimal control of stochastic nonlinear systems with continuous state variables. We prove in Theorem 1 that symmetries of the state dynamics and stage costs imply analogous symmetries of the optimal cost-to-go function and the optimal policy. This allows for the optimal cost-to-go function to be computed in a reduced coordinate system that depends on fewer state variables. In contrast with work on symmetry reduction for MDPs, our results apply to systems with continuous state variables. It is often preferable to identify symmetries in the continuous state space, where symmetries can be identified based on conservation laws or problem geometry, rather than attempting to identify symmetries of a MDP that results from discretizing the continuous state space. In contrast with approximate dynamic programming methods, symmetries lead to a reduction that is exact and does not require any approximation to the optimal cost function or the optimal policy.

We illustrate how this reduction technique can be applied on a problem in magnetic resonance imaging (MRI). We have developed a discrete-time model of spin dynamics as a tool for designing optimized flip angle sequences for MRI fingerprinting, an emerging technology with significant clinical potential [21], [22]. Our model possesses six continuous states, with a rotational symmetry about the axis of the main magnetic field. By exploiting symmetry we can reduce the evaluation of the optimal cost function to a grid of only five dimensions. This significantly reduces the computational burden of performing the dynamic programming algorithm.

This paper is organized as follows. In Section III we introduce notation and provide background information on dynamic programming for optimal control. In Section III we define a notion of symmetry for a control system and prove that control system symmetries induce symmetries of the optimal cost function and optimal control policy. Then in Section IV] we apply symmetry reduction to compute the solution of an optimal control problem arising in dynamic MRI acquisition. Code to reproduce the computational results in this paper is available at https://github.com/ maidens/ACC-2017

\section{BACKGRound: DynAmic Programming FOR OPTIMAL CONTROL OF STOCHASTIC SySTEMS}

We consider a discrete-time dynamical system

$$
x_{k+1}=f_{k}\left(x_{k}, u_{k}, w_{k}\right), \quad k=0,1, \ldots, N-1
$$

where $x_{k} \in \mathcal{X} \subseteq \mathbb{R}^{n}$ is the system state, $u_{k} \in \mathcal{U} \subseteq \mathbb{R}^{m}$ is the control variable to be chosen at time $k, w_{k} \in \mathcal{W} \subseteq \mathbb{R}^{\ell}$ are independent continuous random variables each with density 
$p_{k}$, and $N \in \mathbb{Z}_{+}$is a finite control horizon. Associated with this system is an additive cost function

$$
g_{N}\left(x_{N}\right)+\sum_{k=0}^{N-1} g_{k}\left(x_{k}, u_{k}, w_{k}\right)
$$

that we wish to minimize through our choice of $u_{k}$. We define a control system to be a tuple $\mathcal{S}=(\mathcal{X}, \mathcal{U}, \mathcal{W}, p, f, g, N)$ where $p=\prod_{k=0}^{N-1} p_{k}$ is the joint density of the random variables $w_{k}$.

We consider a class of control policies $\pi=$ $\left\{\mu_{0}, \ldots, \mu_{N-1}\right\}$ where $\mu_{k}: \mathcal{X} \rightarrow \mathcal{U}$ maps observed states to admissible control inputs. Given an initial state $x_{0}$ and a control policy $\pi$, we define the expected cost under this policy as

$$
J_{\pi}\left(x_{0}\right)=\mathbb{E}\left[g_{N}\left(x_{N}\right)+\sum_{k=0}^{N-1} g_{k}\left(x_{k}, \mu_{k}\left(x_{k}\right), w_{k}\right)\right] .
$$

An optimal policy $\pi^{*}$ is defined as one that minimizes the expected cost:

$$
J_{\pi^{*}}\left(x_{0}\right)=\min _{\pi \in \Pi} J_{\pi}\left(x_{0}\right)
$$

where $\Pi$ denotes the set of all admissible control policies. The optimal cost function, denoted $J^{*}\left(x_{0}\right)$, is defined to be the expected cost corresponding to an optimal policy.

An optimal policy $\pi^{*}$ and the optimal cost function $J^{*}$ can be computed using the dynamic programming algorithm. We quote the following result due to Bellman from [23]:

Propostion 1 (Dynamic Programming): For every initial state $x_{0}$, the optimal cost $J^{*}\left(x_{0}\right)$ of the basic problem is equal to $J_{0}\left(x_{0}\right)$, given by the last step of the following algorithm, which proceeds backward in time from period $N-1$ to period 0 :

$$
\begin{aligned}
& J_{N}\left(x_{N}\right)=g_{N}\left(x_{N}\right) \\
& J_{k}\left(x_{k}\right)=\min _{u_{k} \in \mathcal{U}} \mathbb{E}\left[g_{k}\left(x_{k}, u_{k}, w_{k}\right)+J_{k+1}\left(f_{k}\left(x_{k}, u_{k}, w_{k}\right)\right)\right] \\
& \quad k=0,1, \ldots, N-1,
\end{aligned}
$$

where the expectation is taken with respect to the probability distribution of $w_{k}$. Furthermore, if $u_{k}^{*}=\mu_{k}^{*}\left(x_{k}\right)$ minimizes the right hand side of (1) for each $x_{k}$ and $k$, then the policy $\pi^{*}=\left\{\mu_{0}^{*}, \ldots, \mu_{N-1}^{*}\right\}$ is optimal.

\section{SyMmetry REDUCTION}

In order to combat the "curse of dimensionality," we describe a method to reduce the system's dimension by exploiting symmetries in the dynamics and stage costs. We model symmetries based on transformations of the control system.

Definition 1 (Transformation group): A transformation group on $\mathcal{X} \times \mathcal{U} \times \mathcal{W}$ is set of tuples $h_{\alpha}=\left(\phi_{\alpha}, \chi_{\alpha}, \psi_{\alpha}\right)$ parametrized by elements $\alpha$ of a group $\mathcal{G}$ such that the functions $\phi_{\alpha}: \mathcal{X} \rightarrow \mathcal{X}, \chi_{\alpha}: \mathcal{U} \rightarrow \mathcal{U}$ and $\psi_{\alpha}: \mathcal{W} \rightarrow \mathcal{W}$ are all $C^{1}$ diffeomorphisms and they satisfy:

- $\phi_{e}(x)=x, \chi_{e}(u)=u, \psi_{e}(w)=w$ when $e$ is the identity of the group $\mathcal{G}$ and
- $\phi_{a * b}(x)=\phi_{a} \circ \phi_{b}(x), \chi_{a * b}(u)=\chi_{a} \circ \chi_{b}(u), \psi_{a * b}(x)=$ $\psi_{a} \circ \psi_{b}(x)$ for all $a, b \in \mathcal{G}$ where $*$ denotes the group operation and $\circ$ denotes function composition.

To simplify notation we will sometimes suppress the subscripts $\alpha$.

Definition 2 (Invariant control system with invariant costs): A control system $\mathcal{S}$ is $\mathcal{G}$-invariant with $\mathcal{G}$-invariant costs if for all $\alpha \in \mathcal{G}, x_{k} \in \mathcal{X}, u_{k} \in \mathcal{U}$ and $w_{k} \in \mathcal{W}$ we have:

$$
\begin{gathered}
\phi^{-1} \circ f_{k}\left(\phi\left(x_{k}\right), \chi\left(u_{k}\right), \psi\left(w_{k}\right)\right)=f_{k}\left(x_{k}, u_{k}, w_{k}\right), \\
k=0,1, \ldots, N-1 \\
g_{k}\left(\phi\left(x_{k}\right), \chi\left(u_{k}\right), \psi\left(w_{k}\right)\right)=g_{k}\left(x_{k}, u_{k}, w_{k}\right), \\
k=0,1, \ldots, N-1, \\
g_{N}\left(\phi\left(x_{N}\right)\right)=g_{N}\left(x_{N}\right), \text { and } \\
p_{k}\left(\psi\left(w_{k}\right)\right)\left|\operatorname{det} D \psi\left(w_{k}\right)\right|=p_{k}\left(w_{k}\right) \\
k=0,1, \ldots, N-1 .
\end{gathered}
$$

where $D \psi$ denotes the differential of $\psi$. Invariances with respect to a transformation group correspond to symmetries of a control system. Similar definitions have appeared in [24]-[26] for symmetries of deterministic systems.

Theorem 1 (Symmetries of the optimal cost and policy): Let $\mathcal{G}$ be a group and let $\mathcal{S}$ be a $\mathcal{G}$-invariant control system with $\mathcal{G}$-invariant costs. Then the optimal cost functions $J_{k}\left(x_{0}\right)$ satisfy the symmetry relations

$$
J_{k}=J_{k} \circ \phi_{\alpha}
$$

for any $k=0, \ldots, N$ and any $\alpha \in \mathcal{G}$. Furthermore, if $\pi^{*}=$ $\left\{\mu_{0}^{*}, \ldots, \mu_{N-1}^{*}\right\}$ is an optimal policy then so is $\tilde{\pi}^{*}:=\left\{\chi_{\alpha} \circ\right.$ $\left.\mu_{0}^{*} \circ \phi_{\alpha}^{-1}, \ldots, \chi_{\alpha} \circ \mu_{N-1}^{*} \circ \phi_{\alpha}^{-1}\right\}$ for any $\alpha \in \mathcal{G}$.

This theorem readily implies the problem can be reduced, as all states along an orbit of $\mathcal{G}$ are equivalent in terms of cost, and that there are equivalence classes of optimal policies. So it suffices to only consider the cost corresponding to a single representative of each equivalence class, and to find a single representative of the optimal policy within each class.

Proof: First, note that

$$
J_{N}\left(x_{N}\right)=g_{N}\left(x_{N}\right)=g_{N}\left(\phi\left(x_{N}\right)\right)=J_{N}\left(\phi\left(x_{N}\right)\right) .
$$

Now, suppose that for some $k \in\{0, \ldots, N-1\}$ we have $J_{k+1}\left(x_{k+1}\right)=J_{k+1}\left(\phi\left(x_{k+1}\right)\right)$ for all $x_{k+1} \in \mathcal{X}$. Then for any $x_{k} \in X$, and $u_{k} \in \mathcal{U}$ we have

$$
\begin{aligned}
& \mathbb{E}\left[g_{k}\left(x_{k}, u_{k}, w_{k}\right)+J_{k+1}\left(f_{k}\left(x_{k}, u_{k}, w_{k}\right)\right)\right] \\
& =\int_{\mathcal{W}}\left[g_{k}\left(x_{k}, u_{k}, w_{k}\right)+J_{k+1}\left(f_{k}\left(x_{k}, u_{k}, w_{k}\right)\right)\right] p_{k}\left(w_{k}\right) d w_{k} \\
& =\int_{\mathcal{W}}\left[g_{k}\left(\phi\left(x_{k}\right), \chi\left(u_{k}\right), \psi\left(w_{k}\right)\right)+J_{k+1}\left(\phi^{-1} \circ f_{k}\left(\phi\left(x_{k}\right), \chi\left(u_{k}\right), \psi\left(w_{k}\right)\right)\right)\right] p_{k}\left(w_{k}\right) d w_{k} \\
& =\int_{\mathcal{W}}\left[g_{k}\left(\phi\left(x_{k}\right), \chi\left(u_{k}\right), \psi\left(w_{k}\right)\right)+J_{k+1}\left(f_{k}\left(\phi\left(x_{k}\right), \chi\left(u_{k}\right), \psi\left(w_{k}\right)\right)\right)\right] p_{k}\left(w_{k}\right) d w_{k} \\
& =\int_{\mathcal{W}}\left[g_{k}\left(\phi\left(x_{k}\right), \chi\left(u_{k}\right), \psi\left(w_{k}\right)\right)+J_{k+1}\left(f_{k}\left(\phi\left(x_{k}\right), \chi\left(u_{k}\right), \psi\left(w_{k}\right)\right)\right)\right] p_{k}\left(\psi\left(w_{k}\right)\right)\left|\operatorname{det} D \psi\left(x_{k}\right)\right| d w_{k}
\end{aligned}
$$




$$
\begin{aligned}
= & \int_{\psi(\mathcal{W})}\left[g_{k}\left(\phi\left(x_{k}\right), \chi\left(u_{k}\right), \tilde{w}_{k}\right)+J_{k+1}\left(f_{k}\left(\phi\left(x_{k}\right), \chi\left(u_{k}\right), \tilde{w}_{k}\right)\right)\right] p_{k}\left(\tilde{w}_{k}\right) d \tilde{w}_{k} \\
= & \int_{\mathcal{W}}\left[g_{k}\left(\phi\left(x_{k}\right), \chi\left(u_{k}\right), w_{k}\right)+J_{k+1}\left(f_{k}\left(\phi\left(x_{k}\right), \chi\left(u_{k}\right), w_{k}\right)\right)\right] p_{k}\left(w_{k}\right) d w_{k} \\
= & \mathbb{E}\left[g_{k}\left(\phi\left(x_{k}\right), \chi\left(u_{k}\right), w_{k}\right)+J_{k+1}\left(f_{k}\left(\phi\left(x_{k}\right), \chi\left(u_{k}\right), w_{k}\right)\right)\right]
\end{aligned}
$$

Therefore,

$$
\begin{aligned}
J_{k}\left(x_{k}\right) & =\min _{u_{k} \in \mathcal{U}} \mathbb{E}\left[g_{k}\left(x_{k}, u_{k}, w_{k}\right)+J_{k+1}\left(f_{k}\left(x_{k}, u_{k}, w_{k}\right)\right)\right] \\
& =\min _{u_{k} \in \mathcal{U}} \mathbb{E}\left[g_{k}\left(\phi\left(x_{k}\right), \chi\left(u_{k}\right), w_{k}\right)+J_{k+1}\left(f_{k}\left(\phi\left(x_{k}\right), \chi\left(u_{k}\right), w_{k}\right)\right)\right] \\
& =\min _{\tilde{u}_{k} \in \chi(\mathcal{U})} \mathbb{E}\left[g_{k}\left(\phi\left(x_{k}\right), \tilde{u}_{k}, w_{k}\right)+J_{k+1}\left(f_{k}\left(\phi\left(x_{k}\right), \tilde{u}_{k}, w_{k}\right)\right)\right] \\
& =J_{k}\left(\phi\left(x_{k}\right)\right) .
\end{aligned}
$$

Thus $J^{*}=J^{*} \circ \phi$. Now, if $\pi^{*}=\left\{\mu_{0}^{*}, \ldots, \mu_{N-1}^{*}\right\}$ is an optimal policy and we denote $\tilde{x}_{k}=\phi\left(x_{k}\right)$ then for any $k \in$ $\{0, \ldots, N-1\}$ we have

$$
\begin{aligned}
J_{k}\left(\tilde{x}_{k}\right) & =J_{k}\left(x_{k}\right) \\
& =\mathbb{E}\left[g\left(x_{k}, \mu_{k}^{*}\left(x_{k}\right), w_{k}\right)+J_{k+1}\left(f_{k}\left(x_{k}, \mu_{k}^{*}\left(x_{k}\right), w_{k}\right)\right)\right] \\
& =\mathbb{E}\left[g_{k}\left(\phi\left(x_{k}\right), \chi\left(\mu_{k}^{*}\left(x_{k}\right)\right), w_{k}\right)+J_{k+1}\left(f_{k}\left(\phi\left(x_{k}\right), \chi\left(\mu_{k}^{*}\left(x_{k}\right)\right), w_{k}\right)\right)\right] \\
& =\mathbb{E}\left[g_{k}\left(\phi\left(x_{k}\right), \chi \circ \mu_{k}^{*} \circ \phi^{-1}\left(\phi\left(x_{k}\right)\right), w_{k}\right)+J_{k+1}\left(f_{k}\left(\phi\left(x_{k}\right), \chi \circ \mu_{k}^{*} \circ \phi^{-1}\left(\phi\left(x_{k}\right)\right), w_{k}\right)\right)\right] \\
& =\mathbb{E}\left[g_{k}\left(\tilde{x}_{k}, \chi \circ \mu_{k}^{*} \circ \phi^{-1}\left(\tilde{x}_{k}\right), w_{k}\right)+J_{k+1}\left(f_{k}\left(\tilde{x}_{k}, \chi \circ \mu_{k}^{*} \circ \phi^{-1}\left(\tilde{x}_{k}\right), w_{k}\right)\right)\right]
\end{aligned}
$$

Thus $\tilde{\pi}^{*}:=\left\{\chi \circ \mu_{0}^{*} \circ \phi^{-1}, \ldots, \chi \circ \mu_{N-1}^{*} \circ \phi^{-1}\right\}$ is an optimal policy.

\section{APPLICATION: MRI FINGERPRINTING}

Magnetic resonance imaging (MRI) has traditionally focused on acquisition sequences that are static, in the sense that sequences typically wait for magnetization to return to equilibrium between acquisitions. Recently, researchers have demonstrated promising results based on dynamic acquisition sequences, in which spins are continuously excited by a sequence of random input pulses, without allowing the system to return to equilibrium between pulses. Model parameters corresponding to $T_{1}$ and $T_{2}$ relaxation, offresonance and spin density are then estimated from the sequence of acquired data. This technique, termed magnetic resonance fingerprinting (MRF), has been shown to increase the sensitivity, specificity and speed of magnetic resonance studies [21], [22].

We believe that this technique could be further improved by replacing randomized input pulse sequences with sequences that have been optimized for informativeness about model parameters. To this end, we present a model of MR spin dynamics that describes the measured data as a function of $T_{1}$ and $T_{2}$ relaxation rates and the sequence of radiofrequency (RF) input pulses, used to excite the spins.

We model the spin dynamics via the equations

$$
\mathbf{x}_{k+1}=U_{k}\left[\begin{array}{ccc}
\theta_{2} & 0 & 0 \\
0 & \theta_{2} & 0 \\
0 & 0 & \theta_{1}
\end{array}\right] \mathbf{x}_{k}+\left[\begin{array}{c}
0 \\
0 \\
1-\theta_{1}
\end{array}\right]
$$

where the states $x_{1, k}$ and $x_{2, k}$ describe the transverse magnetization (orthogonal to the applied magnetic field) and $x_{3, k}$ describes the longitudinal magnetization (parallel to the applied magnetic field). To simplify the presentation, offresonance is neglected in this model. Control inputs $U_{k} \in$ $\mathrm{SO}(3)$ describe flip angles corresponding to RF excitation pulses that rotate the state about the origin. Between acquisitions, transverse magnetization decays according to the parameter $\theta_{2}=e^{-\Delta t / T_{2}}$ and the longitudinal magnetization recovers to equilibrium (normalized such that the equilibrium is $x_{0}=\left[\begin{array}{lll}0 & 0 & 1\end{array}\right]^{T}$ ) according to the parameter $\theta_{1}=e^{-\Delta t / T_{1}}$ where $\Delta t$ is the sampling interval.

We assume that data are acquired immediately following the RF pulse, allowing us to make a noisy measurement of the transverse magnetization magnitude. We also assume that the measured data are described by a multivariate Gaussian random variable

$$
\mathbf{y}_{k}=\left[\begin{array}{lll}
1 & 0 & 0 \\
0 & 1 & 0
\end{array}\right] \mathbf{x}_{k}+v_{k}
$$

where $v_{k}$ is a zero-mean Gaussian noise with covariance $\left[\begin{array}{ll}\gamma & 0 \\ 0 & \gamma\end{array}\right]$. This model results from a time discretization of the Bloch equations [27], [28] under a time scale separation assumption that specifies that the RF excitation pulses act on a much faster time scale than the relaxation time constants $T_{1}$ and $T_{2}$. A simplified two-state version of this model was considered in [29], where the transverse magnetization was modelled using a single state describing the magnitude of $\left[x_{1, k}, x_{2, k}\right]^{T}$.

We see from the model (2) that magnetization in the transverse direction decays while magnetization in the longitudinal direction grows. However only the transverse component of the magnetization can be measured. Thus there is a tradeoff between making measurements (which leads to loss of magnetization) and magnetization recovery. This is the tradeoff that we hope to manage through the optimal design of an input sequence $U_{k}$.

We wish to quantify the informativeness of an acquisition sequence based on the information about the $T_{1}$ relaxation parameter $\theta_{1}$ that is contained in the resulting data set. More formally, we wish to choose $U_{k} \in \mathrm{SO}(3)$ to maximize the Fisher information about $\theta_{1}$ contained in the joint distribution of $Y=\left(\mathbf{y}_{0}, \ldots, \mathbf{y}_{N}\right)$. The Fisher information $\mathcal{I}$ can be expressed as a quadratic function of the sensitivities of $\mathbf{x}_{k}$ with respect to $\theta_{1}$ :

$$
\mathcal{I}=\sum_{k=0}^{N} \frac{\partial}{\partial \theta_{1}} \mathbf{x}_{k}^{T}\left[\begin{array}{ccc}
1 / \gamma & 0 & 0 \\
0 & 1 / \gamma & 0 \\
0 & 0 & 0
\end{array}\right] \frac{\partial}{\partial \theta_{1}} \mathbf{x}_{k}
$$

where the sensitivities $\frac{\partial}{\partial \theta_{1}} \mathbf{x}_{k}$ satisfy the following sensitivity equations:

$\frac{\partial}{\partial \theta_{1}} \mathbf{x}_{k+1}=U_{k}\left[\begin{array}{ccc}\theta_{2} & 0 & 0 \\ 0 & \theta_{2} & 0 \\ 0 & 0 & \theta_{1}\end{array}\right] \frac{\partial}{\partial \theta_{1}} \mathbf{x}_{k}+U_{k}\left[\begin{array}{ccc}0 & 0 & 0 \\ 0 & 0 & 0 \\ 0 & 0 & 1\end{array}\right] \mathbf{x}_{k}+\left[\begin{array}{c}0 \\ 0 \\ -1\end{array}\right]$

It should be noted that for system (2), the objective function $\mathcal{I}$ has many local optima as a function of the input 


$$
\begin{aligned}
& f_{k}\left(x_{k}, U_{k}, w_{k}\right)==\left[\begin{array}{cccccc}
U_{k} & & 0 & 0 & 0 \\
0 & 0 & 0 & & 0 & 0 \\
0 & 0 & 0 & & U_{k} \\
0 & 0 & 0 & &
\end{array}\right]\left[\begin{array}{cccccc}
\theta_{2} & 0 & 0 & 0 & 0 & 0 \\
0 & \theta_{2} & 0 & 0 & 0 & 0 \\
0 & 0 & \theta_{1} & 0 & 0 & 0 \\
0 & 0 & 0 & \theta_{2} & 0 & 0 \\
0 & 0 & 0 & 0 & \theta_{2} & 0 \\
0 & 0 & 1 & 0 & 0 & \theta_{1}
\end{array}\right] x_{k}+\left[\begin{array}{c}
0 \\
0 \\
1-\theta_{1} \\
0 \\
0 \\
-1
\end{array}\right] \\
& g_{k}\left(x_{k}, U_{k}, w_{k}\right)=-x_{k}^{T}\left[\begin{array}{cccccc}
0 & 0 & 0 & 0 & 0 & 0 \\
0 & 0 & 0 & 0 & 0 & 0 \\
0 & 0 & 0 & 0 & 0 & 0 \\
0 & 0 & 0 & \frac{1}{\gamma} & 0 & 0 \\
0 & 0 & 0 & 0 & \frac{1}{\gamma} & 0 \\
0 & 0 & 0 & 0 & 0 & 0
\end{array}\right] x_{k}
\end{aligned}
$$

sequence $U_{k}$. Thus, in contrast with [30], [31] which consider optimal experiment design for hyperpolarized MRI problems, for this model, local search methods provide little insight into what acquisition sequences are good. In contrast with the MRI model presented in [32], where global optimal experiment design heuristics are developed for linear dynamical systems, in this model the decision variables $U_{k}$ multiply the state vector $\mathbf{x}_{k}$, making the output $\mathbf{y}_{k}$ a nonlinear function of the sequence $U=\left(U_{0}, \ldots U_{k-1}\right)$. Thus we must use dynamic programming to find a solution.

\section{A. Model}

To present this problem in the formalism we have introduced, we define an augmented state vector

$$
x_{k}=\left[\begin{array}{c}
\mathbf{x}_{k} \\
\frac{\partial}{\partial \theta_{1}} \mathbf{x}_{k}
\end{array}\right] \in \mathbb{R}^{6} .
$$

We can write the dynamics of the augmented state as a control system with $f$ and $g$ defined in Equation (3). This system has a one-dimensional group of symmetries defined by

$$
\begin{aligned}
\phi_{\alpha}\left(x_{k}\right) & =\left[\begin{array}{cccccc}
\cos (\alpha) & -\sin (\alpha) & 0 & 0 & 0 & 0 \\
\sin (\alpha) & \cos (\alpha) & 0 & 0 & 0 & 0 \\
0 & 0 & 1 & 0 & 0 & 0 \\
0 & 0 & 0 & \cos (\alpha) & -\sin (\alpha) & 0 \\
0 & 0 & 0 & \sin (\alpha) & \cos (\alpha) & 0 \\
0 & 0 & 0 & 0 & 0 & 1
\end{array}\right] x_{k} \\
\chi_{\alpha}\left(U_{k}\right) & =\left[\begin{array}{ccc}
\cos (\alpha) & -\sin (\alpha) & 0 \\
\sin (\alpha) & \cos (\alpha) & 0 \\
0 & 0 & 1
\end{array}\right] U_{k}\left[\begin{array}{ccc}
\cos (\alpha) & \sin (\alpha) & 0 \\
-\sin (\alpha) & \cos (\alpha) & 0 \\
0 & 0 & 1
\end{array}\right] \\
\psi_{\alpha}\left(w_{k}\right) & =w_{k}
\end{aligned}
$$

for any $\alpha \in \mathbb{R} / 2 \pi \mathbb{Z}$.

\section{B. Dynamic programming in reduced coordinates}

From Theorem 1 we know that the optimal cost functions $J_{k}$ are symmetric with respect to the rotation $\phi_{\alpha}$. Thus all the information about $J_{k}$ can be obtained via its value at points satisfying $x_{1, k} \geq 0, x_{2, k}=0$. Indeed for a general point $x_{k} \in \mathbb{R}^{6}$, the value of $J_{k}\left(x_{k}\right)$ is equal to $J_{k}\left(\bar{x}_{k}\right)$ where

$$
\bar{x}_{k}=\left[\begin{array}{c}
r \\
0 \\
x_{3, k} \\
{\left[\begin{array}{ccc}
\cos (-\alpha) & -\sin (-\alpha) & 0 \\
\sin (-\alpha) & \cos (-\alpha) & 0 \\
0 & 0 & 1
\end{array}\right]\left[\begin{array}{l}
x_{4, k} \\
x_{5, k} \\
x_{6, k}
\end{array}\right]}
\end{array}\right]
$$

with

$$
\begin{aligned}
& \alpha=\operatorname{atan} 2\left(x_{2, k}, x_{1, k}\right) \\
& r=\sqrt{x_{1, k}^{2}+x_{2, k}^{2}} .
\end{aligned}
$$

This significantly reduces the number of grid points at which $J_{k}$ must be evaluated during each step of the dynamic programming algorithm (1).

The problem's symmetry also enables us to reduce the computational burden associated with each evaluation of $J_{k}$. Each evaluation requires the computation of a minimum over the set of three-dimensional rotations $\mathcal{U}=\mathrm{SO}(3)$. We parametrize this set as

$$
U_{k}(\alpha, \beta, \delta)=R_{z}(\alpha) R_{y}(\beta) R_{x}(\delta)
$$

where $-\pi \leq \alpha \leq \pi,-\pi \leq \beta \leq \pi$, and $0 \leq \delta \leq \pi$, and where $R_{z}, R_{y}$, and $R_{x}$ represent rotation matrices about the $\mathrm{z}, \mathrm{y}$, and $\mathrm{x}$ axes respectively. Due to the symmetry of $J_{k+1}$ we know that the choice of $\alpha$ has no effect on the value of

$$
J_{k+1}\left(f_{k}\left(x_{k}, U_{k}(\alpha, \beta, \delta), w_{k}\right)\right) .
$$

This enables us to also reduce the size of the space of inputs.

\section{Results}

Computations were performed on an Amazon Web Services EC2 m4.4xlarge instance with 16 cores and 64GB shared memory. The code was written in the Julia language and parallelized to allow evaluation of $J_{k}$ in parallel across grid points. The implementation is publicly available at https://github.com/maidens/ACC-2017. The complexity of dynamic programming based on the full model (3) is compared against the symmetry-exploiting reduced method described in Section IV-B The results are given 


\begin{tabular}{|l|c|c|c|}
\hline & input grid size & state grid size & runtime \\
\hline Full model & $16 \times 16 \times 8$ & $5 \times 5 \times 5 \times 7 \times 7 \times 7$ & $12662.0 \mathrm{~s}$ \\
\hline Reduced model & $16 \times 8$ & $3 \times 5 \times 7 \times 7 \times 7$ & $170.0 \mathrm{~s}$ \\
\hline
\end{tabular}

TABLE I: Comparison of dynamic programming complexity in original coordinate system against the reduced coordinate system that exploits symmetry.

in Table I. We see that the symmetry reduction leads to a speedup by a factor of approximately $75 \times$ for this problem.

Optimal input and state trajectories for the full (unreduced) model corresponding to the initial condition at the equilibrium $x_{0}=\left[\begin{array}{lllllll}0 & 0 & 1 & 0 & 0 & 0\end{array}\right]^{T}$ are plotted in Figures 1. 2, and 3. The symmetry of the model implies that there are multiple equivalent optimal trajectories. Due to this ambiguity, the optimum that is found by the unreduced dynamic programming algorithm rotates unnecessarily about the $x_{3}$ axis. This shortcoming is eliminated in the reduced algorithm.

Optimal input and state trajectories corresponding to the reduced model described in Section IV-B are shown in Figures 4 and 5 . Comparing Figure 3 with Figure 5 we see that the optimal trajectories computed in the full and reduced coordinates agree relatively well, though there is some error due to the coarse state space grid that was used.

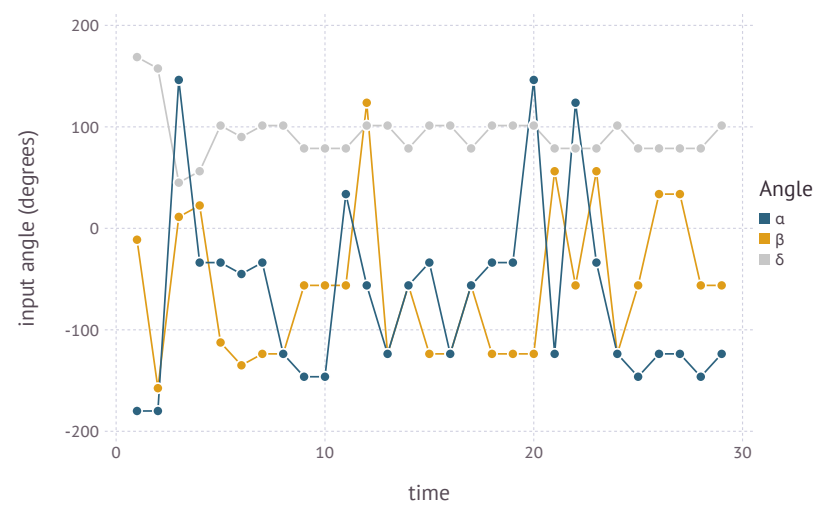

Fig. 1: Optimal input sequence for the full MRI model (3).

\section{CONCLUSION}

We have introduced a method for reducing the complexity of dynamic programming iterations for control systems with symmetries. We prove that control system symmetries induce corresponding symmetries of the optimal cost function and optimal policies. The symmetry of the optimal cost function enables dynamic programming iterations to be performed over a reduced space, leading to significant computational savings. We illustrate this method using a model developed for designing optimized flip angle sequences for MRI fingerprinting. For this model, the symmetry reduction results in an algorithm that runs 75 times faster than dynamic programming based on a naive gridding of the state space.

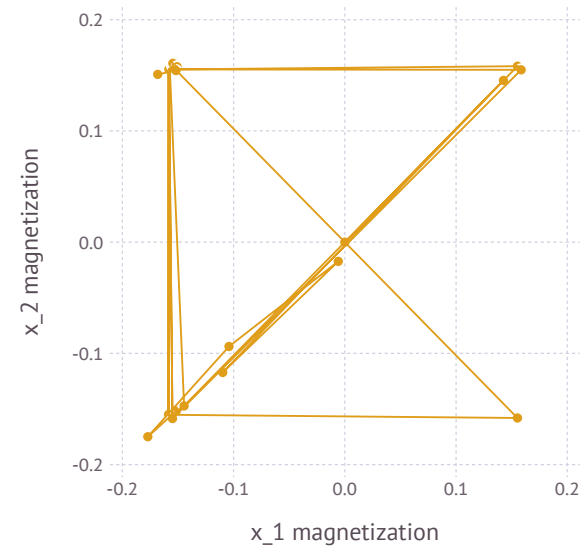

Fig. 2: Optimal state sequence for the full MRI model (3) projected onto the $x_{1}$ and $x_{2}$ coordinates.

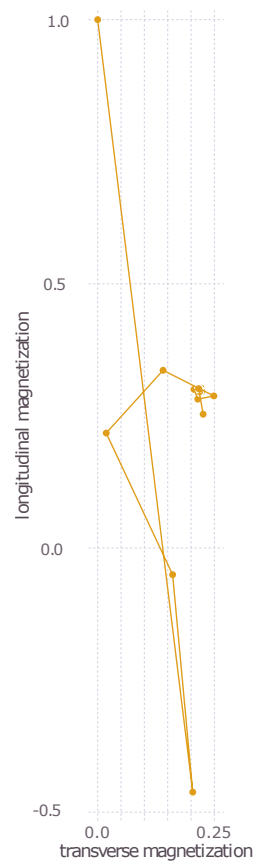

Fig. 3: Optimal state sequence for the full MRI model (3) plotted in coordinates $x_{\text {transverse }}=\sqrt{x_{1, k}^{2}+x_{2, k}^{2}} x_{\text {longitudinal }}=$ $x_{3, k}$.

\section{ACKNOWLEDGEMENTS}

We wish to thank an anonymous reviewer whose feedback improved the statement of Definition 2 and the proof of Theorem 1. 


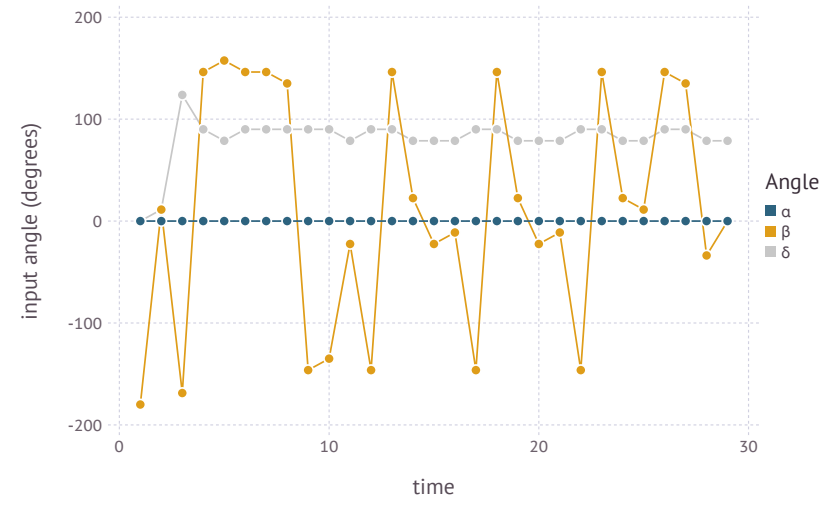

Fig. 4: Optimal input sequence for the reduced MRI model.

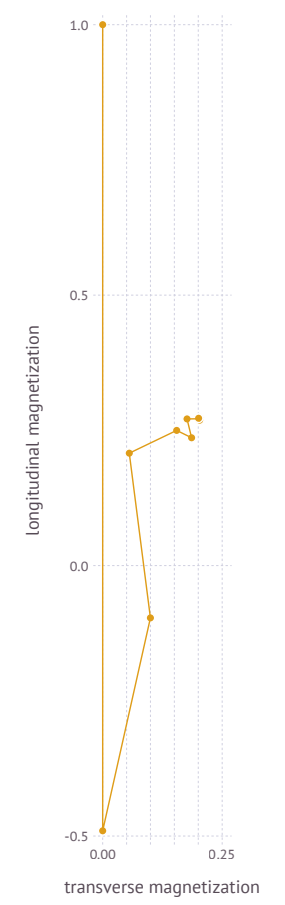

Fig. 5: Optimal state sequence for the reduced MRI model plotted in coordinates $x_{\text {transverse }}=r x_{\text {longitudinal }}=x_{3, k}$.

\section{REFERENCES}

[1] R. Bellman, Dynamic Programming. Princeton University Press, 1957.

[2] R. Bellman and S. Dreyfus, "Functional approximations and dynamic programming," Mathematical Tables and Other Aids to Computation, vol. 13 , no. 68 , pp. $247-251,1959$

[3] D. P. Bertsekas, Dynamic Programming and Optimal Control, Volume II: Approximate Dynamic Programming, 4th ed. Athena Scientific, 2012.

[4] W. B. Powell, Approximate Dynamic Programming: Solving the Curses of Dimensionality. Wiley-Interscience, 2007.

[5] —_, "Perspectives of approximate dynamic programming," Annals of Operations Research, vol. 241, no. 1, pp. 319-356, 2016.

[6] P. A. Clarksonz and E. L. Mansfield, "Symmetry reductions and exact solutions of a class of nonlinear heat equations," Physica D: Nonlinear Phenomena, vol. 70, no. 3, pp. 250 - 288, 1994.

[7] G. Bluman and S. Kumei, Symmetries and Differential Equations, ser. Applied Mathematical Sciences. Springer, 2013.
[8] E. A. Emerson and A. P. Sistla, "Symmetry and model checking," Formal Methods in System Design, vol. 9, no. 1, pp. 105-131, 1996.

[9] M. Kwiatkowska, G. Norman, and D. Parker, Symmetry Reduction for Probabilistic Model Checking. Springer, 2006, pp. 234-248.

[10] A. M. Bloch, P. S. Krishnaprasad, J. E. Marsden, and R. M. Murray, "Nonholonomic mechanical systems with symmetry," Arch. Rational Mech. Anal., vol. 136, pp. 21-99, 1996.

[11] F. Bullo and R. M. Murray, "Tracking for fully actuated mechanical systems: a geometric framework," Automatica, vol. 35, no. 1, pp. 17 34, 1999.

[12] A. Barrau and S. Bonnabel, "Intrinsic filtering on Lie groups with applications to attitude estimation," IEEE Trans. Automatic Control, vol. 60 , no. 2 , pp. 436 - 449, 2015.

[13] _ - "The invariant extended Kalman filter as a stable observer," IEEE Trans. Automatic Control, 2017.

[14] A. Rahmani, M. Ji, M. Mesbahi, and M. Egerstedt, "Controllability of multi-agent systems from a graph-theoretic perspective," SIAM Journal on Control and Optimization, vol. 48, no. 1, pp. 162-186, 2009.

[15] M. Arcak, C. Meissen, and A. Packard, "Symmetry reduction," in Networks of Dissipative Systems: Compositional Certification of Stability, Performance, and Safety. Springer, 2016, pp. 55-62.

[16] A. Rufino Ferreira, C. Meissen, M. Arcak, and A. Packard, "Symmetry reduction for performance certification of interconnected systems," IEEE Transactions on Control of Networked Systems, 2017.

[17] J. Grizzle and S. Marcus, "Optimal control of systems possessing symmetries," IEEE Transactions on Automatic Control, vol. 29, no. 11, pp. 1037-1040, 1984.

[18] T. Ohsawa, "Symmetry reduction of optimal control systems and principal connections," SIAM Journal on Control and Optimization, vol. 51, no. 1, pp. 96-120, 2013.

[19] M. Zinkevich and T. Balch, "Symmetry in Markov decision processes and its implications for single agent and multi agent learning," in In Proceedings of the 18th International Conference on Machine Learning. Morgan Kaufmann, 2001, pp. 632-640.

[20] S. M. Narayanamurthy and B. Ravindran, "Efficiently exploiting symmetries in real time dynamic programming," in Proceedings of the 20th International Joint Conference on Aritifical Intelligence, ser. IJCAI'07. Morgan Kaufmann, 2007, pp. 2556-2561.

[21] D. Ma, V. Gulani, N. Seiberlich, K. Liu, J. L. Sunshine, J. L. Duerk, and M. A. Griswold, "Magnetic resonance fingerprinting," Nature, vol. 495, no. 7440, pp. 187-192, 032013.

[22] M. Davies, G. Puy, P. Vandergheynst, and Y. Wiaux, "A compressed sensing framework for magnetic resonance fingerprinting," SIAM Journal on Imaging Sciences, vol. 7, no. 4, pp. 2623-2656, 2014.

[23] D. P. Bertsekas, Dynamic Programming and Optimal Control, Volume I, 3rd ed. Athena Scientific, 2005.

[24] P. Martin, P. Rouchon, and J. Rudolph, "Invariant tracking," ESAIM: Control, Optimisation and Calculus of Variations, vol. 10, no. 1, pp. $1-13,2004$

[25] B. Jakubczyk, "Symmetries of nonlinear control systems and their symbols," in Canadian Math. Conf. Proceed, vol. 25, 1998, pp. 183198 .

[26] W. Respondek and I. A. Tall, "Nonlinearizable single-input control systems do not admit stationary symmetries," Systems \& Control Letters, vol. 46, no. 1, pp. 1-16, 2002

[27] F. Bloch, "Nuclear induction," Phys. Rev., vol. 70, pp. 460-474, 1946

[28] D. G. Nishimura, Principles of Magnetic Resonance Imaging. Lulu, 2010

[29] J. Maidens, A. Packard, and M. Arcak, "Parallel dynamic programming for optimal experiment design in nonlinear systems," in Proceedings of the Conference on Decision and Control (CDC), 2016, pp. 2894-2899.

[30] J. Maidens, P. E. Z. Larson, and M. Arcak, "Optimal experiment design for physiological parameter estimation using hyperpolarized carbon-13 magnetic resonance imaging," in Proceedings of the American Control Conference (ACC), 2015, pp. 5770-5775.

[31] J. Maidens, J. W. Gordon, M. Arcak, and P. E. Z. Larson, "Optimizing flip angles for metabolic rate estimation in hyperpolarized carbon-13 MRI," IEEE Transactions on Medical Imaging, vol. 35, pp. 24032412, 2016.

[32] J. Maidens and M. Arcak, "Semidefinite relaxations in optimal experiment design with application to substrate injection for hyperpolarized MRI," in Proceedings of the American Control Conference (ACC), 2016, pp. 2023-2028. 